\title{
IMPLEMENTACIÓN A NIVEL UNIVERSITARIO DEL CURSO INTEL EDUCAR PARA EL FUTURO: SISTEMATIZACIÓN DE LA EXPERIENCIA EN LA FACULTAD DE EDUCACIÓN DE LA UNIVERSIDAD DE COSTA RICA
}

Flora Eugenia Salas Madriz

\author{
Recibido 15-VII-2002 • Aceptado 8-X-2002
}

\begin{abstract}
Resumen: La Facultad de Educación de la Universidad de Costa Rica, consciente del impacto e importancia que están adquiriendo las tecnologías educativas digitales en el ámbito educativo a nivel nacional y mundial, ha creado el Programa Tecnologías Educativas Avanzadas (PROTEA) para el desarrollo y la investigación en este campo. En este artículo se sistematiza la experiencia de nueve docentes de la Universidad de Costa Rica, que colaboraron en la adecuación para el nivel universitario del curso Intel Educar para el Futuro, impartido por la Fundación Omar Dengo. Éste está orientado a educadores de primaria y secundaria, y su propósito es poner a disposición de los educadores las herramientas del Office ${ }^{\circledR}$ de Microsoft ${ }^{\circledR}$ para desarrollar los procesos de enseñanza. Debido a las diferencias de fondo y forma entre la educación primaria y secundaria, y la universitaria, se hizo necesario revisar el manual y la metodología a fin de adaptarlos a las necesidades de la docencia universitaria y de la formación de formadores. En este sentido, la experiencia y colaboración de los docentes participantes fue determinante para lograr que el curso alcance hoy sus objetivos de poner estas herramientas al servicio de la docencia universitaria.
\end{abstract}

Palabras clave: Tecnología Educativa, Capacitación Docente, Investigación Cualitativa, herramientas Digitales.

\section{Antecedentes}

Los profundos cambios económicos, políticos, sociales y culturales que vivimos a nivel global en la última década del siglo XX, ya perfilaban un impacto de consecuencias inéditas en todos los niveles educativos. Conceptos como el de "educación permanente" y el de "educación para la vida de por vida", atravesaron de principio a fin los supuestos tradicionales de la educación a nivel mundial, para poner de manifiesto, ante las instancias correspondientes, tanto gubernamentales como privadas, la necesidad irrenunciable e impostergable de ofrecer respuestas viables y rápidas a las nuevas demandas de educación en todo el planeta.

Esta realidad ha sido evidente en Costa Rica, donde se ha dado un incremento en la demanda por educación, a lo largo de la última década. La educación privada, por ejemplo, ha tenido un impresionante desarrollo en el país, ofreciendo alternativas de educación en todos los niveles, a distintos sectores de la población que presionados por el contexto económico y social de hoy hacen importantes inversiones de tiempo y dinero en la adquisición de educación que les permita acceder con éxito a la compleja trama de competitividad laboral y social propias de la lógica del capitalismo tardío. Esta realidad ha convertido 
la educación en una actividad económica altamente rentable que hace necesarias instancias de control y revisión permanentes, a fin de que realmente cumpla con el papel que le ha sido asignado por los sistemas económico, político y social actuales.

La educación pública, por su parte, ha tenido que responder no sólo al incremento en la demanda de educación de calidad en todos los niveles, sino también a la presión por el aceso a alternativas novedosas de formación que ofrezcan respuestas a los diversos requerimientos y necesidades sectoriales, empresariales, gubernamentales y personales que, a su vez, se vuelven más específicos. Dentro de esta compleja y diversa red de señales para producir y orientar los cambios, los entes encargados de la educación pública han debido enfrentar, en el corto y mediano plazos, el reto de introducir profundas transformaciones epistemológicas, teóricas, administrativas, de infraestructura y de tecnología. Estas transformaciones, por supuesto, han propiciado esfuerzos de distinta naturaleza, con el propósito de solventar las deficiencias encontradas en estas áreas. El proceso ha sido difícil y complejo, y todavía quedan muchas tareas pendientes, lo que no significa que algunos de estos vacíos empiecen a ser llenados para dar paso a una educación pública de calidad, accesible, oportuna y pertinente.

Como parte de este esfuerzo por el logro de una educación pública de calidad, oportuna y pertinente, el Programa de Tecnologías Educativas Avanzadas (PROTEA), de la Facultad de Educación de la Universidad de Costa Rica, empieza sus funciones en el año 2001 con la participación de representantes de las Escuelas de Administración Educativa, Educación Física, Orientación y Educación Especial, Bibliotecología, y de Formación Docente, así como con el apoyo del Instituto de Investigaciones para el Mejoramiento de la Educación Costarricense (IIMEC). Este proyecto nace con el objetivo principal de crear las condiciones de infraestructura y de cultura organizacional y docente, necesarias para la incorporación de tecnologías educativas digitales en la formación de docentes y estudiantes de las distintas carreras impartidas en la Facultad de Educación. Por esta razón, entre sus principales estrategias estaba crear vínculos con instituciones públicas que tuvieran lineamientos en esta dirección. Dada la experiencia acumulada en este campo, se entró en negociaciones con la Fundación Omar Dengo (FOD), que ya había implementado con éxito el programa Intel Educar para el futuro, con una cobertura nacional, financiado y auspiciado a nivel pedagógico e informático por Componentes Intel de Costa Rica (Intel), como parte de un programa mayor de cooperación en distintos países alrededor del mundo.

La respuesta de la FOD no sólo fue positiva, sino que se pudo ampliar el marco de acción original del proyecto, orientado a la formación de educadores de primaria y secundaria, con la anuencia y colaboración de Componentes Intel de Costa Rica, a la docencia universitaria y a la formación de formadores. Este es, precisamente, el proceso que pasamos a exponer, a fin de que esta experiencia pueda ser capitalizada por otras instituciones de educación superior, tanto a nivel nacional como internacional.

\section{La implementación del curso Intel educar para el futuro en la Facultad de Educación de la Universidad de Costa Rica}

Durante el año 2001 se inició el proyecto de capacitación a docentes y a estudiantes de la Facultad de Educación de la Universidad de Costa Rica, en las herramientas informáticas de Office, a través del convenio con la Fundación Omar Dengo y con Componentes Intel de Costa Rica. Para tal efecto, se introdujo el curso Intel Educar para el Futuro, tal y como había 
sido diseñado originalmente y fueron capacitados 40 estudiantes y 25 docentes.

Con base en la experiencia acumulada, los docentes que recibieron el curso externaron una serie de inquietudes que fueron atendidas por el Comité Ejecutivo de PROTEA. Entre la más importante estaba el señalamiento de que el curso no respondía a las necesidades e intereses de los docentes y estudiantes universitarios. Con el propósito de resolver este problema, se propuso una experiencia piloto que permitiera una adecuación del curso para el nivel universitario, como se explica a continuación.

\subsection{Sistematización de la experiencia piloto para la modificación del manual}

En el mes de enero del 2002, se programó un curso para docentes universitarios en el laboratorio de cómputo de la Facultad de Educación de la Universidad de Costa Rica. Este curso tuvo un doble propósito: capacitar al personal docente y evaluar y sistematizar la experiencia, a fin de que sirviera como base para la adecuación al nivel universitario del manual Intel educar para el futuro, orientado originalmente, como se mencionó, a educadores de primaria y secundaria. Por esta razón y debido a las diferencias de fondo y forma entre la educación primaria y secundaria y la universitaria, se hizo necesario revisar el manual y la metodología, a fin de adaptarlos a las necesidades de la docencia universitaria y de la formación de formadores en el campo de la educación.

Con la participación de nueve docentes, entre los cuales hubo un miembro de la Comisión del Programa de Tecnologías Educativas Avanzadas, a quien se le encargó realizar el proceso de sistematización de la experiencia, se dio un curso intensivo del 14 al 23 de enero del 2002, que permitió, a través de la discusión y el análisis en grupo así como de las experiencias personales de los participantes relatadas en el portafolio del curso, elaborar el primer borrador del manual para ser revisado por la $F O D$ y por los personeros de Componentes Intel de Costa Rica, para su posterior aprobación e implementación a nivel universitario.

\subsubsection{Metodología de trabajo}

$\mathrm{Al}$ iniciar el curso se expuso a los estudiantes la idea de trabajar con ellos en una experiencia piloto, así como los objetivos y metodología de la misma. Todos estuvieron anuentes a colaborar y a participar en las actividades que se les propuso. Participaron en el curso:

\section{Ana Cecilia Hernández Rodríguez Flora Eugenia Salas Madriz María Eugenia Jenkis Alvarado María Marta Camacho Alvarez Marjorie Solano Siles Pilar Carvajal Mora Randall Figueroa Mata Roxana María González Bonilla Victoria González García}

Como metodología de trabajo para la sistematización de la experiencia, se partió de un enfoque cualitativo, ya que interesaba conocer la percepción, intereses y motivaciones de los participantes, así como su propia experiencia en el uso del manual. La observación participante permitió conocer cómo los docentes en proceso de capacitación vivían la experiencia de aprendizaje a través del manual Intel educar para el futuro. También se utilizó la autoobservación, ya que esta técnica permite alcanzar los niveles más altos de comprensión del sentido de las acciones de los sujetos, pues certeza y comprensión del sentido son los fundamentos de la validez de la auto-observación (Delgado, 1998: 154). Todo lo anterior, con el propósito de que el nuevo manual para docentes y estudiantes universitarios, realmente cumpliera con los 
requerimientos y expectativas generales de esta población.

Otra técnica utilizada fue la discusión en grupo de temas de interés, derivados de la experiencia en el curso, que permitió conocer temores, vacíos cognoscitivos, expectativas y mitos, en torno a la utilización de las computadoras en distintos ámbitos de la vida académica y personal. Las necesidades sentidas, así como las preguntas generadoras, permitieron detectar aspectos estructurales, organizativos y culturales en la Universidad de Costa Rica, que limitan los espacios para la incorporación masiva, eficiente y eficaz de las tecnologías educativas digitales. Entre las más importantes destaca, precisamente, la ausencia de un programa permanente de capacitación y actualización en esta área, donde instancias como el Departamento de Docencia Universitaria y el Programa de Tecnologías Educativas Avanzadas de la Facultad de Educación, deben jugar un papel protagónico. Problemas de infraestructura informática, así como deficiencias de orden administrativo y de gestión docente, fueron también señaladas como limitaciones para una adecuada implementación de la tecnología educativa digital en las actividades cotidianas de los docentes.

Todos los participantes coincidieron en que las tecnologías digitales ofrecen un amplio campo de acción para la docencia universitaria, que debe ser incorporado lo antes posible como parte del quehacer propio de profesores y estudiantes en la Universidad de Costa Rica, siempre y cuando se haga con base en una reflexión paradigmática, pedagógica y didáctica, que realmente rompa con el esquema tradicional de impartir lecciones, ya que, de lo contrario, se corre el riesgo de "seguir haciendo lo mismo con tecnologías educativas costosas".

Entre los aspectos que destaca, como puede observarse, está el que la implementación de tecnologías educativas digitales tiene implícita una elaboración teórico-pedagógica de fondo que no puede ser obviada. Resulta indispensable para que estas tecnologías realmente contribuyan a un cambio significativo en la calidad de nuestro sistema educativo, la superación de esquemas tradicionalmente conductistas 0 , peor aún, de esquemas confusos en donde posiciones epistemológicamente opuestas -anticonstructivismo, constructivismo-, están presentes simultáneamente en procesos de enseñanzaaprendizaje.

Seguidamente, se muestra una síntesis y análisis de la información dada por los participantes del curso, que fue organizada de la siguiente manera:

\section{a. Mitos y temores.}

b. Expectativas sobre el curso y sobre las tecnologías educativas digitales.

c. Formas de aprendizaje y paradigmas educativos.

d. El propio aprendizaje y el curso.

e. Recomendaciones y sugerencias para mejorar el curso.

\section{a. Mitos y temores}

A través de la discusión en grupo, se pudo encontrar que entre las principales preocupaciones que externaron las docentes, está el hecho de que normalmente se asume que la tecnología digital es en sí misma y por sí misma significativa a nivel de aprendizaje. Consideran que este ha sido el principal mito en torno a esta tecnología, y que ello ha llevado a un uso equivocado de la misma. Por esta razón, señalan que se ha continuado dentro de los esquemas pedagógicos tradicionales, a pesar del esfuerzo realizado a nivel institucional por equipar a la Universidad de Costa Rica con una infraestructura informática destinada específicamente a la docencia: 


\section{Ana Cecilia Hernández (Portafolio):}

Personalmente considero que las aplicaciones de la computación al campo educativo son pocas y muy limitadas, por ejemplo, la más empleada, multimedia con PowerPoint, reafirma el esquema tradicional de "dar lecciones", aunque éste sea preparado y presentado por los estudiantes. Hay que seguir trabajando pero en la línea de materiales educativos que superen el conductismo.

\section{María Marta Camacho (Portafolio):}

Se considera vital que el estudiante universitario conozca y aplique nuevas herramientas tecnológicas. Crear espacio para usarlas y para analizarlas es labor fundamental. No sólo se trata de saber aplicar una herramienta, se trata de discutir los logros, usos, e influencia que estas herramientas pueden ejercer en nuestra labor y en nuestro mundo actual.

Destacan las participantes que sin una reflexión profunda y consecuente con las demandas actuales sobre la educación en general, no será posible aprovechar la tecnología educativa digital en toda su dimensión. Esta reflexión supone, a su vez, un replanteamiento del enfoque pedagógico tradicional, así como el reto de implementar, de manera auténtica y coherente, el constructivismo en el aula. Todas las participantes coincidieron en que todavía se está muy lejos de una práctica real del constructivismo, ya que aún se encuentran presentes distorsiones de corte tradicional y conductista. Ahora bien, no se trata de descalificar de manera radical al conductismo, ya que este enfoque aún es válido y puede ser muy útil en ciertas circunstancias en donde el aprendizaje programado sea la meta. Sin embargo, apuntan que dadas las características del mundo actual, en donde el cambio y la toma de decisiones se han acelerado en magnitudes sin precedentes, un modelo educativo que no promueva destrezas y habilidades en los niveles cognitivo y socio-afectivo, difícilmente será el adecuado para la preparación de profesionales que se desempeñarán en el siglo XXI.
En cuanto a los temores personales, a través de la discusión en grupo, se señaló que una buena parte de docentes universitarios pertenece a generaciones que quedaron inmersas en la transición de la docencia y las tecnologías educativas tradicionales, a la docencia apoyada en el paradigma constructivista y en las tecnologías educativas digitales. Esto ha tenido como consecuencia que sea necesario efectuar un proceso de alfabetización informática, una reforma pedagógica, y una capacitación en las tecnologías educativas digitales, para cerrar la brecha y la ambigüedad que viven estas personas, entre el esquema tradicional y las nuevas tendencias pedagógicas y didácticas. Por tal motivo, muchos docentes se encuentran en una paradógica situación: desean asimilar y aplicar las nuevas corrientes educativas y las nuevas tecnologías, pero temen enfrentarse al hecho de que desconocen aspectos básicos de las computadoras, tanto en lo que respecta a hardware como a software. Incluso, señalan las participantes el hecho de que muchos docentes aún no saben como manipular adecuadamente el mouse o cómo digitar. Esta realidad implica frustración, negación y resistencia por parte de los docentes, que sienten como una amenaza la incorporación de las nuevas tecnologías educativas digitales en su práctica profesional y académica:

\section{María Eugenia Jenkis (Portafolio)}

Es interesante observar y sentir como la adquisición de destrezas mejora la autoestima.

Mi autoestima ha mejorado, mi conocimiento se ha incrementado, se han ampliado mis relaciones interpersonales y los deseos de aprender tienen nuevos horizontes.

Por otro lado, es de analizar el hecho de que los logros continuos puedan generar dependencia de la computadora.

Además, se pudo discutir y analizar en grupo el hecho de que el ingresar en el 
marco de posibilidades que abre la tecnología digital en el ámbito educativo, genera el temor de que se "invisibilice" al docente. El temor por el borramiento del papel que juega el educador en el proceso enseñanzaaprendizaje, es uno de los principales factores de resistencia. Se cree que la tecnología va a llegar a bastarse a sí misma para enseñar; idea que, por demás, no considera el hecho de que las computadoras no pueden hacer absolutamente nada por sí mismas. Fue necesario reflexionar sobre el hecho de que detrás de todo producto informático siempre se encuentra la inteligencia, creatividad e intereses de una persona o de un grupo de personas.

No es la primera vez que la humanidad enfrenta el desafío de asimilar los cambios que introducen en su vida sus propias producciones científico-técnicas. Con la Revolución Industrial, el fantasma de la omnipotencia de la máquina se hizo presente. Contra las máquinas que emularon músculo humano se levantó una fuerte resistencia, ya que los trabajadores consideraban que éstas les iban a despojar de su trabajo; un ejemplo clásico de esta resistencia lo constituyen los movimientos de trabajadores agrícolas en Europa, a principios del siglo XX, contra la consechadora mecánica. Esto fue cierto sólo parcialmente ya que a partir de la incorporación de las máquinas, las personas tuvieron acceso a elevar el tiempo destinado a su educación lo que, a su vez, permitió que se potenciaran la inteligencia y creatividad humanas. Las demandas por educación se han ido elevando conforme se han incrementado las necesidades de personas más educadas y formadas en todos los ámbitos del saber. Obviamente, este proceso ha sido y es sumamente complejo, ya que supone un acelerado desarrollo del modo de producción capitalista, que lleva implícitas las asimetrías de clase y la desigual distribución de la riqueza y de las oportunidades de desarrollo.

Si bien es cierto no todos los sectores de la población tienen acceso en nuestros días a una educación más larga y desarrollada, es innegable su tendencia a ampliarse y alcanzar sectores sociales otrora excluidos. De hecho, uno de los retos del siglo XXI es, precisamente, atender las necesidades y características de esta creciente demanda por educación que se extiende a nivel global, propiciada y movilizada, paradójicamente, por el mismo desarrollo del capitalismo en su fase tardía.

Con la incorporación de máquinas capaces de emular cerebro humano, la omnipotencia de la máquina ha cobrado nuevas fuerzas y tomado otras características. La amenaza se cierne ahora sobre los trabajadores intelectuales. Sin embargo, al igual que en el caso de las máquinas que emularon músculo humano a partir del siglo XIX, este temor es infundado, ya que lo que se abre son nuevos espacios para el desarrollo de la inteligencia y la creatividad humanas, destinados a potenciar los procesos educativos y cognoscitivos, y a reducir la cantidad de tiempo y dinero que se debe invertir para aprender y para producir conocimiento. Sin embargo, también nos enfrentamos con el hecho de que no todos los seres humanos en el planeta tienen acceso a estas nuevas oportunidades. Se calcula que sólo un $10 \%$ de la población mundial tiene hoy acceso a computadoras, y éstas viven en su mayoría en países desarrollados. Por lo tanto, el problema no deviene de las computadoras en sí, sino de las asimetrías mundiales de la distribución de la riqueza.

Como hemos podido ver hasta ahora, los mitos y temores sobre la introducción de la computadora en los procesos de enseñanza-aprendizaje, obedecen al impacto y cambios que esta tecnología produce a nivel personal, profesional, económico, institucional y social. La superación de estos temores está, como pudo observarse a lo largo del curso, en la asimiliación y comprensión de estas tecnologías, que sólo se logran a través de una sensibilización y contacto directos con las posibilidades y limitaciones reales de las computadoras; es 
decir, la única forma de desmitificar a la máquina es eliminando su "apariencia" de omnipotencia, para dimensionarla en su lugar de herramienta al servicio del ser humano. Por esta razón, es tan importante la capacitación oportuna y permanente en el uso y aplicaciones de la tecnología en todos los campos, particularmente, en el de la educación. Al respecto, es interesante ver como la capacitación ofrecida a las docentes permitió reducir la resistencia y el malestar, y reemplazarlos por satisfacción personal y logro profesional:

\section{Victoria González (Portafolio):}

He desarrollado habilidades que me permiten utilizar la tecnología en los procesos pedagógicos en el ejercicio de mi profesión. Me siento más fuerte, más autónoma y más inteligente (el destacado es nuestro). Mi reto personal es lograr esta misma satisfacción en mis estudiantes.

\section{María Marta Camacho (Portafolio):}

Al finalizar el proceso de construcción de una herramienta multimedios, me siento satisfecha por los conocimientos adquiridos tanto en el manejo de Power Point, como en la realización del producto final de mi trabajo. No sólo pude cumplir con las requisitos del trabajo, sino que produje un material muy útil en mi labor docente, en mi desarrollo como persona y como profesional y como medio para el intercambio a nivel social (comunicación de masas).

Otro aspecto que es necesario resaltar es que en el área de la socio-afectividad me ha permitido sentirme muy motivada, haciendo un trabajo que considero útil e invirtiendo mi tiempo en algo que me agrada y al que voy a sacarle provecho.

Me siento también más inteligente, con más poder y menos estafada (el destacado es nuestro), ya que ahora tengo conocimientos nuevos para realizar cosas que antes tenía que pagar porque no sabía cómo realizarlas por mis propios medios.

El empoderamiento y la seguridad personal se logran a través de la apropiación cognitiva y subjetiva de un saber, habilidad o destreza particulares. Como puede observarse en los comentarios de las participantes, la sensación de logro y la desmitificación de la tecnología al ponerla al servicio de los intereses propios, contribuyen al mejoramiento profesional y a la práctica docente, redundando este proceso, inevitablemente, en el mejoramiento en la calidad de la enseñanza.

\section{b. Expectativas sobre el curso y sobre las tecnologías educativas computacionales}

Entre las principales espectivas sobre el curso y la aplicabilidad de las tecnologías educativas computacionales, destaca la necesidad de desarrollar la capacidad para propiciar espacios de enseñanzaaprendizaje. Además, resultó interesante el proceso de apropiación de las herramientas informáticas para el desarrollo personal, académico y profesional. Al respecto, veamos algunos de los comentarios de las docentes que participaron en el curso:

\section{Marjorie Solano (Portafolio):}

Estoy muy satisfecha pues he obtenido un buen aprendizaje en el desarrollo del curso. Sobre todo, la aplicación que puedo darle en el desarrollo docente.

He desarrollado habilidades que me permiten utilizar la tecnología en los procesos pedagógicos en el ejercicio de mi profesión.

\section{Victoria González (Portafolio):}

Ha sido una experiencia activa, dinámica, de mucha construcción social, y de interacción tanto profesional como de aprendizaje y a nivel humano.

\section{María Marta Camacho (Portafolio):}

Considero que esta experiencia es muy útil para mi vida personal y profesional. Ha sido todo un éxito y un motor de motivación y de cambio.

Conforme el curso iba avanzando, las participantes se fueron involucrando en aplicaciones específicas de las herramientas 
informáticas para el desarrollo de una propuesta de enseñanza-aprendizaje. La propuesta se derivaba de algún tema de interés personal, que permitiera explorar $\mathrm{y}$, a la vez, elaborar productos específicos en Power Point, Word y Publisher de Microsoft. Esto permitió, además, la discusión pedagógica de cada producto, así como la reflexión, la crítica y la autocrítica, en torno al nivel de logro de los objetivos o metas de aprendizaje propuestos por cada participante. Esta dinámica permitió trascender la herramienta informática, para ubicar los productos al interior de propuestas pedagógicas y didácticas concretas.

Una de las principales demandas de los cursos para docentes impartidos con anterioridad radicó, precisamente, en la necesidad de incorporar una reflexión pedagógica para ubicar los productos elaborados utilizando el medio informático en el ámbito de la docencia universitaria. En este sentido, se considera que esta reflexión fue de suma importancia como complemento del manual del curso, así como para orientar la dirección que éste debía tener para satisfacer los intereses y demandas de la población de interés.

\section{c. Formas de aprendizaje y paradigmas educativos}

Uno de los aspectos del curso que más agrado produjo en las participantes fue la posibilidad de vivir una experiencia de aprendizaje significativo, en la cual se propiciaran el autoaprendizaje, los intereses personales y la creatividad, tanto como el compartir y reflexionar en grupo. El constructivismo fue ampliamente discutido en el curso, no sólo como una posibilidad epistemológica que abre un nuevo campo a la práctica docente, sino también como una situación real vivida en el curso:

\section{Victoria González (Portafolio):}

Para mí es significativo [el aprendizaje] porque lo elegí yo, me gusta, es divertido para mí y aprendo de una manera activa y cuestionadora. Elementos que considero fundamentales para que exista verdadero aprendizaje. En lo que se refiere a las herramientas de computación mi mayor interés se centra en el uso de las posibilidades en Internet y la creación de sitios web. [...]

El trabajo lo hago por mi misma, me propongo una tarea en particular, tomo decisiones en relación con el proceso que deseo realizar. En otras palabras, soy la propia constructora de mi conocimiento y de mis valores.

[...]

Ha sido una experiencia activa, dinámica, de mucha construcción social, y de interacción tanto profesional como de aprendizaje y a nivel humano.

\section{María Marta Camacho (Portafolio):}

Soy constructora de mi propio proceso, soy partícipe de un proceso grupal donde mis ideas y mis acciones son válidas. Soy planificadora de mis acciones y de mi ritmo de trabajo.

[...]

Me encanta saber que trabajo a mi propio ritmo y de acuerdo con mis propios intereses. No es sólo hacer lo que el profesor ha programado, se trata de construir mi propio concepto y ser partícipe de mi propio proceso.

Con base en la vivencia de las participantes, se logró imprimir al curso una lógica constructivista y reflexiva, más que una lógica directiva orientada básicamente al manejo de las herramientas informáticas. El privilegiar los intereses personales permitió que las docentes se sintieran más cómodas y motivadas a apropiarse de las herramientas informáticas como medios para lograr fines de orden personal, académico y profesional.

\section{d. El propio aprendizaje y el curso}

La intención de las docentes que participaron en el curso fue desarrollar sus capacidades y destrezas en el manejo de las herramientas informáticas para la producción de material didáctico.

\section{María Marta Camacho (Portafolio):}

$\mathrm{Al}$ vivir en una era donde la informática cobra tanta influencia, es indispensable mantenerse actualizado. 
Mis expectativas ante esta capacitación se basan en fortalecer conocimientos previos y obtener aprendizajes significativos nuevos.

Para las docentes que tenían un manejo medio de la computadora, el curso resultó bastante útil para la elaboración de propuestas de enseñanza-aprendizaje. Sin embargo, también hubo compañeras que tenían un bajo dominio de la máquina y del software, por lo que su desempeño y avance fueron limitados. Por esta razón, se considera necesario que haya requisitos de ingreso al curso, entre los que se hace indispensable que la persona haya pasado por un proceso previo de alfabetización informática.

A través de la discusión en grupo se pudo determinar que las inquietudes de las docentes en torno al curso se centraban en la posibilidad de encontrar medios para el desarrollo de material didáctico, y no en el uso puramente instrumental de las herramientas informáticas. Si bien es cierto se le dio seguimiento al conocimiento y empleo de las herramientas, el énfasis se puso en el potencial de aprendizaje de cada producto elaborado por las docentes. Con este propósito se hicieron presentaciones de los avances de cada participante, que fueron enriquecidos con los aportes de las compañeras y de la facilitadora del curso.

Otro aspecto significativo detectado a través de la "auto-observación" y de la observación participante, fue la forma como las participantes se fueron familiarizando con las herramientas a través de un "aprender haciendo", que fue propuesto y sostenido por la facilitadora del curso:

\section{Victoria González (Portafolio):}

La facilitadora tuvo características metodológicas importantes: fue flexible, utilizó variedad de recursos didácticos tecnológicos y no tecnológicos, fue abierta, aplicó adecuaciones en lo que se refiere al respeto por nuestro desarrollo y avance individual, nuestros intereses, el seguimiento y monitoreo de nuestros productos, la administración del tiempo durante el curso. Una pedagoga que ayudó brindando herramientas pero sin asumir las cosas por uno.
Otro aspecto a considerar en cuanto a la dinámica del curso son los espacios que se abren para la discusión pedagógica. El poder compartir con compañeras y compañeros de otras unidades académicas enriqueció la discusión y abrió la posibilidad de producir materiales didácticos de una forma novedosa, creativa y crítica:

\section{Victoria González (Portafolio):}

Más que aspectos, hablaría de factores o elementos: el facilitador, los estudiantes, los recursos. Es un grupo de estudiantes con un gran compromiso por desarrollar su propia tarea, por colaborar con los compañeros y compañeras y por dar de sí el mejor producto. Evidentemente, estas lecciones sin los recursos didácticos y materiales necesarios son un absurdo. En este sentido tuvimos una excelente plataforma de trabajo y excelente acompañamiento.

Como parte de la reflexión propiciada en el curso, fue posible replantear aspectos teórico-metodológicos de la práctica de las docentes, lo que permitió delimitar el uso de la tecnología educativa digital a fines didácticos concretos, con la consecuente desmitificación de la misma:

\section{Victoria González (Portafolio):}

Hoy he decidido reestructurar mi trabajo didáctico. Pienso que pensar en unidad didáctica me hace mantenerme en ámbitos escolares menores que la universidad. De manera que sustituiré la UNIDAD DIDÁCTICA por PROGRAMA DEL CURSO SEMESTRAL, dividido en algunas UNIDADES DIDÁCTICAS, seguido de la LíNEA DEL TIEMPO que llamaré CRONOGRAMA y, posteriormente, PLAN DE LECCIÓN. Es el plan de lección el que utilizaré para la presentación multimedia, para la búsqueda de Internet, el diseño de un boletín con Publisher y el sitio web. Así mismo, como una sugerencia, pienso que debe crearse un módulo para los universitarios que trabaje la relación del programa del curso con el resto de la carrera. De manera que se pueda estimular la reflexión en torno a la formación de profesionales en forma integral.

Nótese como la reflexión se llevó a niveles profundos, donde la propuesta de enseñanza-aprendizaje desarrollada por algunas de las participantes permitió, incluso, 
pensar en el necesario anclaje de los productos dentro de una perspectiva global, que incluyera los objetivos del curso que se imparte, y la naturaleza de la carrera misma. Para el cuarto día de capacitación, la herramienta informática y la computadora se habían desmitificado y las participantes se sentían más cómodas, seguras y confiadas al utilizarlas.

Gracias a los procesos de análisis y reflexión y a la orientación dada al curso - tanto en el manual como por la facilitadora-, fue posible que las docentes visualizaran el proceso de enseñanza-aprendizaje y, por ende, la elaboración de sus productos didácticos, desde la perspectiva del estudiante:

\section{María Marta Camacho (Portafolio):}

[Trabajar desde la perspectiva del estudiante] ...es trabajar desde la perspectiva en la que se es sujeto y objeto de su propia formación. Es tomar en cuenta las características de la persona desde una visión integral e integradora, desde un enfoque que toma en cuenta lo que quiero aprender (socioafectividad), qué quiero aprender y cómo (cognitividad), y con cuáles herramientas, procesos, movimientos, interacciones, quiero aprender (psicomotricidad). Todo esto hace que el aprendizaje sea relevante y significativo.

\section{Victoria González (Portafolio):}

He desarrollado habilidades que me permiten utilizar la tecnología en los procesos pedagógicos en el ejercicio de mi profesión. Me siento más fuerte, más autónoma y más inteligente. Mi reto personal es lograr esta misma satisfacción en mis estudiantes.

\section{Marjorie Solano (Portafolio):}

Estoy muy satisfecha pues he obtenido un buen aprendizaje en el desarrollo del curso. Sobre todo, la aplicación que puedo darle en el desarrollo docente.

En términos generales, el curso llenó las expectativas de las participantes, permitiéndoles no sólo empoderarse en cuando a las herramientas informáticas y la computadora propiamente dichas, sino también en lo que respecta a la reflexión que media todo proceso de enseñanza- aprendizaje, independientemente de las estrategias didácticas que se emplee:

\section{María Marta Camacho (Portafolio):}

Felicito a las Instituciones participantes en este proyecto por el servicio social que brindan a la educación costarricense.

Soy constructora de mi propio proceso, soy partícipe de un proceso grupal donde mis ideas y mis acciones son válidas. Soy planificadora de mis acciones y de mi ritmo de trabajo.

Considero que esta experiencia es muy útil para mi vida personal y profesional.

Ha sido todo un éxito y un motor de motivación y de cambio.

\section{Victoria González (Portafolio):}

Ha sido una experiencia activa, dinámica, de mucha construcción social, y de interacción tanto profesional como de aprendizaje y a nivel humano.

Como puede observarse, la experiencia fue positiva y enriquecedora para las participantes, permitiendo, además, con su valiosa colaboración, efectuar una revisión del manual y determinar los lineamientos correctos para la adecuación del curso a los intereses y necesidades de la población universitaria.

\section{e. Recomendaciones y sugerencias para mejorar el curso}

En este apartado pasamos a enumerar y explicar brevemente algunas de las principales recomendaciones y sugerencias dadas por los participantes para mejorar el curso:

1. Realimentación: Desarrollar un programa de educación continua en la Universidad de Costa Rica, que permita a los docentes mantenerse actualizados y realimentar sus experiencias docentes, académicas, personales y profesionales. 
2. Conocer otros profesionales: Propiciar el intercambio de ideas y experiencias con docentes de otras áreas, ya que esto permite ampliar las posibilidades de crecimiento y desarrollo a nivel docente, profesional y personal.

3. Conocer nuevas herramientas: Profundizar en el uso de las herramientas informáticas ya conocidas -Word, Power Point, Publisher-, así como dar acceso al conocimiento de otras como: Excel, Access, y Project, para potenciar y fortalecer la docencia, la investigación y la acción social.

4. Aplicar conocimientos: Propiciar y estimular la producción de materiales de apoyo didáctico a través del uso de recursos informáticos, como una manera eficiente y eficaz de aplicación y transmisión de conocimientos. Al respecto, deben considerarse las ventajas adicionales de que tales productos pueden ser accesados a través de diversos medios, a bajo costo, y en cualquier momento, como, por ejemplo, las páginas web.

5. Generar nuevos aprendizajes: A través de la utilización de recursos informáticos es posible elevar tanto la cantidad como la calidad de los aprendizajes. En este sentido, se trata de aprender de una forma nueva, cosas nuevas.

\section{A manera de conclusión}

Con base en lo dicho hasta aquí, podemos decir que la experiencia fue sumamente enriquecedora, no sólo porque permitió una revisión profunda del manual, sino también porque hizo posible comprender la dirección que debía tomar el curso para ser exitoso y cumplir con la meta de ofrecer a los docentes y estudiantes de la Universidad de Costa Rica, una plataforma sólida y coherente en tecnología educativa digital.

Finalmente, sólo nos resta reconocer la colaboración de las docentes de la Universidad de Costa Rica que llevaron el curso, ya que sin sus aportes este trabajo no hubiera sido posible. A todos ellos, nuestra gratitud.

\section{Referencias bibliográficas}

Delgado, Juan Manuel. Métodos y técnicas cualitativas de investigación en Ciencias Sociales. Madrid: Síntesis, 1998.

Discusión en grupo con la participación de: Ana Cecilia Hernández Rodríguez Flora Eugenia Salas Madriz

María Eugenia Jenkis Alvarado

María Marta Camacho Alvarez

Marjorie Solano Siles

Pilar Carvajal Mora

Randall Figueroa Mata

Roxana María González Bonilla

Victoria González García

Material del portafolio personal aportado por:

María Marta Camacho

Victoria González

María Eugenia Jenkins

Ana Cecilia Hernández

Conducción de la experiencia, observación participante y autobservación:

Flora Eugenia Salas Madriz 
